\title{
EXPERIMENTAL STUDY OF VOLUME CALIBRATION OF MASS WEIGHT WITH HYDROSTATIC WEIGHING
}

\author{
HONG MAN HU \\ Division of Mechanics and Acoustic, National Institute of Metrology, Beijing 100013, P. R. China \\ hmh@nim.ac.cn \\ JIAN WANG, YUE ZHANG, CHANG QING CAI, RUI LIN ZHONG, \\ HONG YAO, JING AN DING \\ Division of Mechanics and Acoustic, National Institute of Metrology, Beijing 100013, P. R. China
}

\begin{abstract}
In this paper, the volumes of mass weights ranging from $500 \mathrm{~g}$ to $1 \mathrm{~g}$ are calibrated with a fused quartz solid density standard using a hydrostatic weighing device developed by Lab of Volume and Density in NIM, China. The stability of liquid temperature is discussed. And also the measurement uncertainty of the densities is evaluated based on the calibration models.
\end{abstract}

Keywords: Hydrostatic weighing method; solid volume calibration; mass standards.

\section{Introduction}

The volume of mass standard is needed to be measured in order to correct the air bouncy during the mass calibration process. Hydrostatic weighing method is one of traditional volume measuring methods with high measuring accuracy. With the development of Avogadro project [1-3], solid density standards are more used for the high repeatability and stability of density value, comparing with the liquid density standards. The volume of mass standard is needed to be measured in order to correct the air bouncy during the mass calibration process. Hydrostatic weighing method is one of traditional volume measuring methods with high measuring accuracy.[4-6]

In this paper, the solid density standard made of fused quartz is used for the volume calibration of mass weights. The volumes of OIML $\mathrm{E}_{1}$ mass weights ranging from $500 \mathrm{~g}$ to $1 \mathrm{~g}$ are tested. And the measurement uncertainty of the densities is also evaluated based on the calibration models.

This is an Open Access article published by World Scientific Publishing Company. It is distributed under the terms of the Creative Commons Attribution 3.0 (CC-BY) License. Further distribution of this work is permitted, provided the original work is properly cited. 


\section{Experimental apparatus}

The experiments are carried out using a hydrostatic weighing device developed by Lab of Volume and Density in NIM, China. Fig. 1 shows the photos of the experimental setup. The weighting capability of the mass comparator (XP50S) is $520 \mathrm{~g}$ with a resolution of $0.01 \mathrm{mg}$. The temperature is monitored by a thermometer with an accuracy of 0.001 Celsius. All the experiments are carried out in n-Tridecane $\left(\mathrm{C}_{13} \mathrm{H}_{28}\right)$, which has a low surface tension. A commercial temperature control system AV7000 (Thomas) is used for the temperature control of the working liquid temperature. The expected temperature control accuracy is $\pm 0.01{ }^{\circ} \mathrm{C}$ with a $1000 \mathrm{~W}$ heat power.

The volumes of test weights ranging from $500 \mathrm{~g}$ to $1 \mathrm{~g}$ are measured with a fused quartz solid density standard showned in Fig. 2, whose density is calibrated using the silicon sphere primary solid density standard.

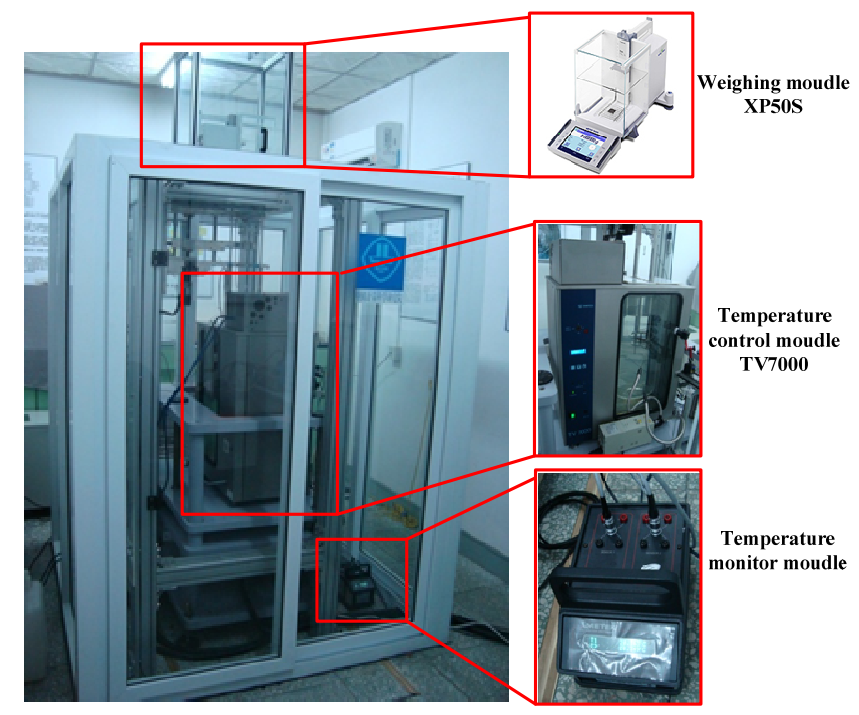

Fig.1 Photos of Experiment setup.

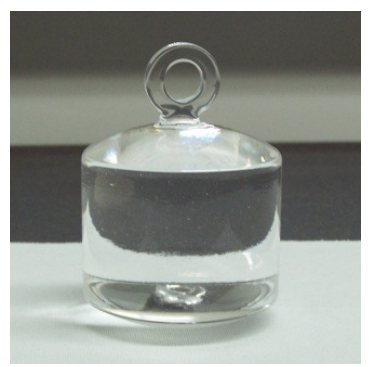

(a)

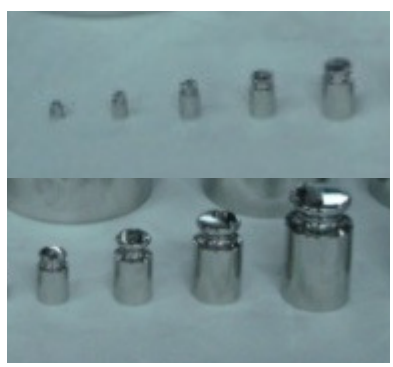

(b)

Fig.2 Photos of fused quartz solid density standard (a) and test weights ranging from $500 \mathrm{~g}$ to $1 \mathrm{~g}$ (b). 


\section{Measurement procedure}

During the volume calibration process, the liquid bouncy to test standards and the fused quartz solid density standard are different. The calibration process of weights' volume mainly includes 3 steps as follows:

Step1: The mass of the fused quartz solid standards in liquid is measured to calculate the density of liquid by compraring with count reference weight 1 in air.

Step2: Calibration the mass value of test weights in air by compraring with count reference weight 2 in air.

Step3: The mass of test standards in liquid is measured subsequently with the same method in step 2 by compraring with count reference weight 3 in air.

The density of pure water can be calculated using the Eq. (1),

$$
\rho_{\mathrm{L}}\left(t_{1}\right)=\frac{m_{\text {quartz }}-m_{\text {weight1 }}+\frac{m_{\text {weight1 }}}{\rho_{\text {weight1 }}}\left[1+\beta_{\text {weight1 }}\left(t_{1}-20\right)\right] \rho_{\text {a1 }}-\Delta I_{1}}{V_{\text {quartz }}\left[1+\beta_{\text {quartz }}\left(t_{1}-20\right)\right]}
$$

in which, $m_{\text {quartz }}$ and $V_{\text {quartz }}$ are the mass and volume of the fused quartz solid density standards. $m_{\text {weight } 1}$ and $\rho_{\text {weight } 1}$ are the mass and density of count reference weight $1 . \beta_{\text {quartz }}$ and $\beta_{\text {weight } 1}$ are the volume expansion coefficient of fused quartz solid standards and count reference weight $1 . \rho_{\mathrm{L}}(t)$ is the liquid density at temperature $t_{1} . \rho_{\mathrm{a} 1}$ is the air density when measurement is proceeded. $\Delta I_{1}$ is the difference of mass comparator's indication between fused quartz density standard in liquid and count reference weight 1 in air.

The volume of test weight can be calculated with the Eq. (2),

$$
V_{\text {test }}=\frac{A-B}{C-D}
$$

in which,

$$
\begin{gathered}
A=m_{\text {weight } 3}+\Delta I_{3}-\frac{m_{\text {weight3 }}}{\rho_{\text {weight3 }}}\left[1+\beta_{\text {weight3 }}\left(t_{3}-20\right)\right] \rho_{\mathrm{a} 3} \\
B=m_{\text {weight2 }}+\Delta I_{2}-\frac{m_{\text {weight2 }}}{\rho_{\text {weight } 2}}\left[1+\beta_{\text {weight2 }}\left(t_{2}-20\right)\right] \rho_{\mathrm{a} 2} \\
C=\left[1+\beta_{\text {test }}\left(t_{2}-20\right)\right] \rho_{a 2} \\
D=\left[1+\beta_{\text {test }}\left(t_{3}-20\right)\right] \rho_{\mathrm{L}}\left(t_{3}\right)
\end{gathered}
$$

in which $m_{\text {weight2 }}$ and $m_{\text {weight3 }}$ are the mass of count reference weight 2 and count reference weight 3. $\rho_{\text {weight2 }}$ and $\rho_{\text {weight3 }}$ are the density of count reference weight 2 and count reference weight 3. $\boldsymbol{V}_{\text {test }}$ are the volume of test weight. $\boldsymbol{\beta}_{\text {test }}, \boldsymbol{\beta}_{\text {test }}, \boldsymbol{\beta}_{\text {test }}$ are the volume expansion coefficient of test weight, count reference weight 2 and count 
reference weight 3. $\rho_{\mathrm{a} 2}$ and $\rho_{\mathrm{a} 3}$ are the air densities. $\Delta I_{2}$ and $\Delta I_{3}$ are the difference of mass comparator's indications between the test weight and count reference weight.

\section{Measurements and results}

Temperature control is a fundamental aspect of the measurement device. The temperature stability is evaluated through a series of test. As shown in Fig. 3(a), under the temperature control of AV7000, the variation of liquid temperature can be limited to 3 $\mathrm{mK}$. And after turn off the the temperature control of AV7000, the liquid temperature can be relatively stable in 30 minutes as shown in Fig. 3(b).

Assume that each parameters are disrelated, the uncertainty of liquid $\rho_{\mathrm{L}}(t)$ can be calculated by taking partial derivative of Eq. (1) with respect to each parameters. And the measurement value and uncertainty values are shown in Table 1 . The combined uncertainty is $0.0016 \mathrm{~kg} / \mathrm{m}^{3}$, and the expanded relative uncertainty is $4.3 \times 10^{-6}(\mathrm{k}=2)$.

(a)

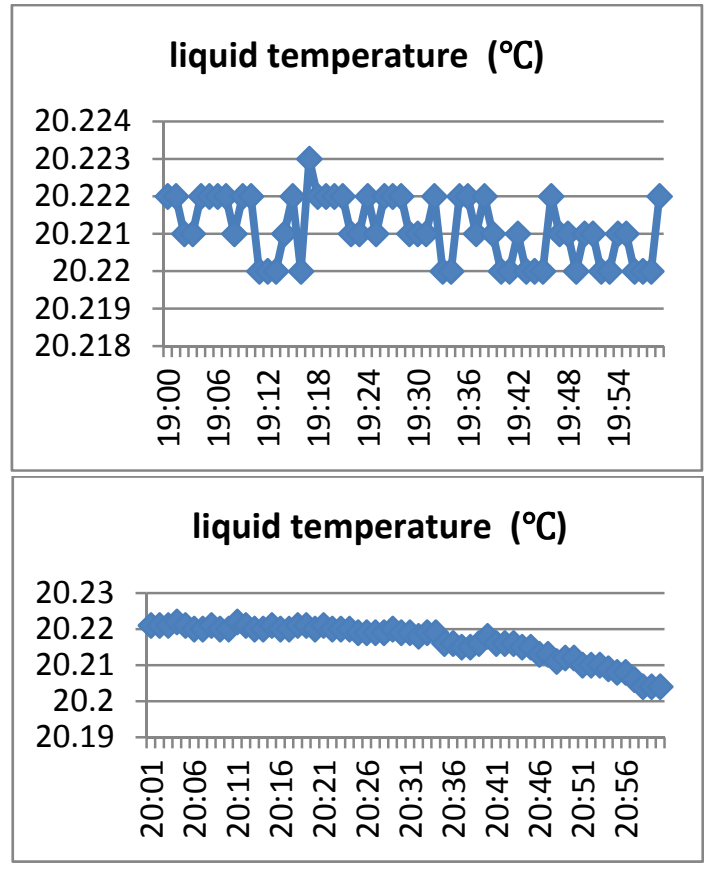

Fig.3 Temperature variation when temperature is on (a) and off (b).

Table 1. Uncertainty budgets for the density of working liquids $\rho_{\mathrm{L}}(t)$.

\begin{tabular}{ccccc}
\hline Source & Value & $\begin{array}{c}\text { Value standard } \\
\text { uncertainty }\end{array}$ & Sensitive coefficient & $\begin{array}{c}\text { Uncertainty } \\
\text { contribution }\end{array}$ \\
\hline$m_{\text {quartz }}$ & $0.32776356 \mathrm{~kg}$ & $0.00000006 \mathrm{~kg}$ & $6720.566 / \mathrm{m}^{3}$ & $0.00041 \mathrm{~kg} / \mathrm{m}^{3}$ \\
$m_{\text {weight1 }}$ & $0.215270178 \mathrm{~kg}$ & $0.00000006 \mathrm{~kg}$ & $0.0117 / \mathrm{m}^{3}$ & $7.1 \times 10^{-10} \mathrm{~kg} / \mathrm{m}^{3}$ \\
$V_{\text {quartz }}$ & $0.000148797 \mathrm{~m}^{3}$ & $0.0000000003 \mathrm{~m}^{3}$ & $-5079437.22 \mathrm{~kg} / \mathrm{m}^{6}$ & $0.00153 \mathrm{~kg} / \mathrm{m}^{3}$ \\
\hline
\end{tabular}


Structural Characteristics of $\mathrm{La}_{2} \mathrm{O}_{3}$ Thin Film Grown on $\mathrm{LaB}_{6}$

\begin{tabular}{ccccc}
\hline$\beta_{\text {quartz }}$ & $0.0000014 /{ }^{\circ} \mathrm{C}$ & $0.000004 /{ }^{\circ} \mathrm{C}$ & $-24.186{ }^{\circ} \mathrm{C} \mathrm{kg} / \mathrm{m}^{3}$ & $9.7 \times 10^{-5} \mathrm{~kg} / \mathrm{m}^{3}$ \\
$\beta_{\text {weight } 1}$ & $0.000046 /{ }^{\circ} \mathrm{C}$ & $0.000004 /{ }^{\circ} \mathrm{C}$ & $1.02 \times 10^{-6}{ }^{\circ} \mathrm{C} \mathrm{kg} / \mathrm{m}^{3}$ & $4.1 \times 10^{-12} \mathrm{~kg} / \mathrm{m}^{3}$ \\
$\rho_{\text {weight } 1}$ & $8000 \mathrm{~kg} / \mathrm{m}^{3}$ & $10 \mathrm{~kg} / \mathrm{m}^{3}$ & $-2.26 \times 10^{-5}$ & $0.000227 \mathrm{~kg} / \mathrm{m}^{3}$ \\
$\rho_{\mathrm{a}}$ & $1.1839 \mathrm{~kg} / \mathrm{m}^{3}$ & $0.0007 \mathrm{~kg} / \mathrm{m}^{3}$ & 0.1809 & $0.00013 \mathrm{~kg} / \mathrm{m}^{3}$ \\
$t_{1}$ & $20.032{ }^{\circ} \mathrm{C}$ & $0.0057{ }^{\circ} \mathrm{C}$ & $0.00106 \mathrm{~kg} / \mathrm{m}^{3} /{ }^{\circ} \mathrm{C}$ & $6.1 \times 10^{-6} \mathrm{~kg} / \mathrm{m}^{3}$ \\
$u\left(\rho_{\mathrm{L}}(t)\right)$ & & & & $0.0016 \mathrm{~kg} / \mathrm{m}^{3}$ \\
$u_{\mathrm{rel}}\left(\rho_{\mathrm{L}}(t)\right)$ & & & & $2.1 \times 10^{-6}$ \\
$U_{\mathrm{rel}}\left(\rho_{\mathrm{L}}(t)\right)$ & & & \\
$(k=2)$ & & & \\
\hline
\end{tabular}

The uncertainty of test weight's volume can be calculated by taking partial derivative of Eq. (2) with respect to each parameter. Take the $10 \mathrm{~g}$ weight for example, the measured volume is $1.25100 \times 10^{-6} \mathrm{~m}^{3}$, the combined uncertainty $\left(u\left(V_{\text {est }}\right)\right)$ is $0.00004 \mathrm{~cm}^{3}$ $(k=2)$. And the measurement value and uncertainty values are shown in Table 2 . The volumes and uncertainty of other weights ranging from $500 \mathrm{~g}$ to $1 \mathrm{~g}$ are shown in Table 3 and Fig. 4.

Table 2. Uncertainty budgets for the volume of the $10 \mathrm{~g}$ weight.

\begin{tabular}{|c|c|c|c|c|}
\hline Source & Value & $\begin{array}{l}\text { Value Standard } \\
\text { uncertainty }\end{array}$ & $\begin{array}{l}\text { Sensitive } \\
\text { coefficient }\end{array}$ & $\begin{array}{l}\text { Uncertianty } \\
\text { contribution }\end{array}$ \\
\hline$u\left(\rho_{\mathrm{L}}\left(t_{3}\right)\right)$ & $756.124994 \mathrm{~kg} / \mathrm{m}^{3}$ & $4.23 \times 10^{-6} \mathrm{~kg} / \mathrm{m}^{3}$ & $1.66 \times 10^{-9} \mathrm{~m}^{6} / \mathrm{kg}$ & $7.01 \times 10^{-9} \mathrm{~cm}^{3}$ \\
\hline $\boldsymbol{m}_{\text {weight2 }}$ & $0.01000002 \mathrm{~kg}$ & $1 \times 10^{-8} \mathrm{~kg}$ & $0.00133 \mathrm{~m}^{3} / \mathrm{kg}$ & $1.33 \times 10^{-5} \mathrm{~cm}^{3}$ \\
\hline $\boldsymbol{m}_{\text {weight3 }}$ & $0.00905470 \mathrm{~kg}$ & $1 \times 10^{-8} \mathrm{~kg}$ & $0.00133 \mathrm{~m}^{3} / \mathrm{kg}$ & $1.33 \times 10^{-5} \mathrm{~cm}^{3}$ \\
\hline$\rho_{\text {weight } 2}$ & $8000 \mathrm{~kg} / \mathrm{m}^{3}$ & $10 \mathrm{~kg} / \mathrm{m}^{3}$ & $2.44 \times 10^{-13} \mathrm{~m}^{6} / \mathrm{kg}$ & $2.44 \times 10^{-6} \mathrm{~cm}^{3}$ \\
\hline$\rho_{\text {weight } 3}$ & $8000 \mathrm{~kg} / \mathrm{m}^{3}$ & $10 \mathrm{~kg} / \mathrm{m}^{3}$ & $2.24 \times 10^{-13} \mathrm{~m}^{6} / \mathrm{kg}$ & $2.24 \times 10^{-6} \mathrm{~cm}^{3}$ \\
\hline $\boldsymbol{\beta}_{\text {test }}$ & $0.000046 /{ }^{\circ} \mathrm{C}$ & $0.000004 /{ }^{\circ} \mathrm{C}$ & $2.79 \times 10^{-7}{ }^{\circ} \mathrm{Cm}^{3}$ & $1.12 \times 10^{-6} \mathrm{~cm}^{3}$ \\
\hline $\boldsymbol{\beta}_{\text {weight2 }}$ & $0.000041 /{ }^{\circ} \mathrm{C}$ & $0.000004 /{ }^{\circ} \mathrm{C}$ & $9.75 \times 10^{-11{ }^{\circ}} \mathrm{C} \mathrm{m}^{3}$ & $3.90 \times 10^{-10} \mathrm{~cm}^{3}$ \\
\hline $\boldsymbol{\beta}_{\text {weight3 }}$ & $0.000043 /{ }^{\circ} \mathrm{C}$ & $0.000004 /{ }^{\circ} \mathrm{C}$ & $3.99 \times 10^{-10}{ }^{\circ} \mathrm{C} \mathrm{m}^{3}$ & $1.60 \times 10^{-9} \mathrm{~cm}^{3}$ \\
\hline$\rho_{\mathrm{a} 2}$ & $1.1772 \mathrm{~kg} / \mathrm{m}^{3}$ & $0.0009 \mathrm{~kg} / \mathrm{m}^{3}$ & $6.11 \times 10^{-13} \mathrm{~m}^{6} / \mathrm{kg}$ & $5.48 \times 10^{-10} \mathrm{~cm}^{3}$ \\
\hline$\rho_{\mathrm{a} 3}$ & $1.1911 \mathrm{~kg} / \mathrm{m}^{3}$ & $0.0009 \mathrm{~kg} / \mathrm{m}^{3}$ & $1.50 \times 10^{-9} \mathrm{~m}^{6} / \mathrm{kg}$ & $1.35 \times 10^{-6} \mathrm{~cm}^{3}$ \\
\hline$t_{2}$ & $20.050^{\circ} \mathrm{C}$ & $0.0057^{\circ} \mathrm{C}$ & $3.31 \times 10^{-17} \mathrm{~m}^{3} /{ }^{\circ} \mathrm{C}$ & $1.99 \times 10^{-13} \mathrm{~cm}^{3}$ \\
\hline$t_{3}$ & $20.223^{\circ} \mathrm{C}$ & $0.0057^{\circ} \mathrm{C}$ & $5.75 \times 10^{-11} \mathrm{~m}^{3} /{ }^{\circ} \mathrm{C}$ & $3.45 \times 10^{-7} \mathrm{~cm}^{3}$ \\
\hline$u\left(V_{\mathrm{est}}\right)$ & & & & $0.00002 \mathrm{~cm}^{3}$ \\
\hline$U\left(V_{\text {est }}\right)(k=2)$ & & & & $0.00004 \mathrm{~cm}^{3}$ \\
\hline
\end{tabular}


Table 3. Uncertainty budgets for the volume of the weights ranging from $500 \mathrm{~g}$ to $1 \mathrm{~g}$.

\begin{tabular}{|c|c|c|c|c|c|c|c|c|}
\hline \multirow{2}{*}{ Source } & \multicolumn{8}{|c|}{ Uncertianty contribution $\left(\mathrm{cm}^{3}\right)$} \\
\hline & $1 \mathrm{~g}$ & $2 \mathrm{~g}$ & $5 \mathrm{~g}$ & $20 \mathrm{~g}$ & $50 \mathrm{~g}$ & $100 \mathrm{~g}$ & $200 \mathrm{~g}$ & $500 \mathrm{~g}$ \\
\hline$u\left(\rho_{\mathrm{L}}\left(t_{3}\right)\right)$ & $7.0 \times 10^{-10}$ & $1.4 \times 10^{-9}$ & $3.5 \times 10^{-9}$ & $1.4 \times 10^{-8}$ & $3.5 \times 10^{-8}$ & $7.0 \times 10^{-8}$ & $1.4 \times 10^{-7}$ & $3.5 \times 10^{-7}$ \\
\hline $\boldsymbol{m}_{\text {weight2 }}$ & $6.6 \times 10^{-6}$ & $8.8 \times 10^{-6}$ & $1.1 \times 10^{-5}$ & $1.8 \times 10^{-5}$ & $2.2 \times 10^{-5}$ & $3.5 \times 10^{-5}$ & $6.6 \times 10^{-5}$ & $1.8 \times 10^{-4}$ \\
\hline $\boldsymbol{m}_{\text {weight3 }}$ & $6.6 \times 10^{-6}$ & $8.8 \times 10^{-6}$ & $1.1 \times 10^{-5}$ & $1.8 \times 10^{-5}$ & $2.2 \times 10^{-5}$ & $3.5 \times 10^{-5}$ & $6.6 \times 10^{-5}$ & $1.7 \times 10^{-4}$ \\
\hline$\rho_{\text {weight } 2}$ & $2.4 \times 10^{-7}$ & $4.9 \times 10^{-7}$ & $1.2 \times 10^{-6}$ & $4.9 \times 10^{-6}$ & $1.2 \times 10^{-5}$ & $2.4 \times 10^{-5}$ & $4.9 \times 10^{-5}$ & $1.2 \times 10^{-4}$ \\
\hline$\rho_{\text {weight3 }}$ & $2.2 \times 10^{-7}$ & $4.5 \times 10^{-7}$ & $1.1 \times 10^{-6}$ & $4.5 \times 10^{-6}$ & $1.1 \times 10^{-5}$ & $2.2 \times 10^{-5}$ & $4.5 \times 10^{-5}$ & $1.1 \times 10^{-4}$ \\
\hline $\boldsymbol{\beta}_{\text {test }}$ & $1.1 \times 10^{-7}$ & $2.2 \times 10^{-7}$ & $5.6 \times 10^{-7}$ & $7.6 \times 10^{-7}$ & $1.1 \times 10^{-6}$ & $9.8 \times 10^{-7}$ & $2.2 \times 10^{-5}$ & $5.6 \times 10^{-5}$ \\
\hline $\boldsymbol{\beta}_{\text {weight2 }}$ & $3.9 \times 10^{-11}$ & $7.8 \times 10^{-11}$ & $1.9 \times 10^{-10}$ & $7.8 \times 10^{-10}$ & $1.9 \times 10^{-9}$ & $3.9 \times 10^{-9}$ & $7.8 \times 10^{-9}$ & $1.9 \times 10^{-8}$ \\
\hline $\boldsymbol{\beta}_{\text {weight3 }}$ & $1.6 \times 10^{-10}$ & $3.1 \times 10^{-10}$ & $7.9 \times 10^{-10}$ & $1.1 \times 10^{-9}$ & $1.6 \times 10^{-9}$ & $1.4 \times 10^{-9}$ & $3.1 \times 10^{-8}$ & $7.9 \times 10^{-8}$ \\
\hline$\rho_{\mathrm{a} 2}$ & $2.4 \times 10^{-10}$ & $1.2 \times 10^{-9}$ & $1.2 \times 10^{-9}$ & $4.8 \times 10^{-9}$ & $1.2 \times 10^{-8}$ & $5.9 \times 10^{-8}$ & $5.4 \times 10^{-8}$ & $7.0 \times 10^{-8}$ \\
\hline$\rho_{\mathrm{a} 3}$ & $1.3 \times 10^{-7}$ & $2.7 \times 10^{-7}$ & $6.7 \times 10^{-7}$ & $2.7 \times 10^{-6}$ & $6.7 \times 10^{-6}$ & $1.3 \times 10^{-5}$ & $2.7 \times 10^{-5}$ & $6.7 \times 10^{-5}$ \\
\hline$t_{2}$ & $8.5 \times 10^{-14}$ & $4.2 \times 10^{-13}$ & $4.3 \times 10^{-13}$ & $1.7 \times 10^{-12}$ & $4.2 \times 10^{-12}$ & $2.1 \times 10^{-11}$ & $2.0 \times 10^{-11}$ & $2.5 \times 10^{-11}$ \\
\hline$t_{3}$ & $3.5 \times 10^{-8}$ & $6.9 \times 10^{-8}$ & $1.7 \times 10^{-7}$ & $6.9 \times 10^{-7}$ & $1.7 \times 10^{-6}$ & $3.5 \times 10^{-6}$ & $6.9 \times 10^{-6}$ & $1.7 \times 10^{-5}$ \\
\hline$u\left(V_{\mathrm{est}}\right)$ & $1.0 \times 10^{-5}$ & $1.3 \times 10^{-5}$ & $1.6 \times 10^{-5}$ & $2.6 \times 10^{-5}$ & $3.6 \times 10^{-5}$ & $6.1 \times 10^{-5}$ & $1.2 \times 10^{-4}$ & $3.1 \times 10^{-4}$ \\
\hline $\begin{array}{c}U\left(V_{\text {est }}\right) \\
(k=2)\end{array}$ & $2 \times 10^{-5}$ & $3 \times 10^{-5}$ & $4 \times 10^{-5}$ & $6 \times 10^{-5}$ & $8 \times 10^{-5}$ & $1.2 \times 10^{-4}$ & $2.4 \times 10^{-4}$ & $6.2 \times 10^{-4}$ \\
\hline
\end{tabular}

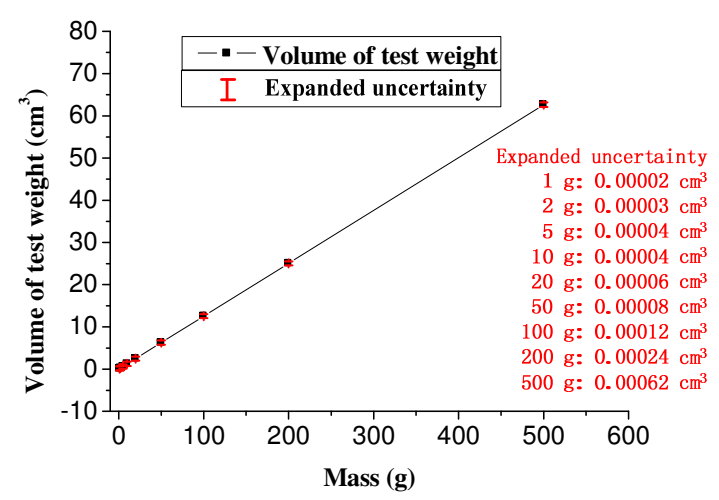

Fig.4 Measurement result and uncertainty evaluation of the volume of test weight ranging from $500 \mathrm{~g} \sim \mathrm{g}$.

\section{Conclusions}

In this paper, the volumes of mass weights ranging from $500 \mathrm{~g}$ to $1 \mathrm{~g}$ are calibrated with a fused quartz solid density standard using a hydrostatic weighing device developed by Lab of Volume and Density in NIM, China. The variation of liquid temperature can be limited under $3 \mathrm{mK}$. The volume measurement expanded uncertainty of the test weights ranging from $500 \mathrm{~g}$ to $1 \mathrm{~g}$ is from $0.00002 \mathrm{~cm}^{3}$ to $0.00064 \mathrm{~cm}^{3}$. 


\section{References}

1. R. A. Nicohus and K. Fujii, Meas Sci Technology. 17, 2527-2539 (2006).

2. K. Fujii, Meas. Sci. Technol. 17, 2551-2559(2006).

3. K.Fujii, Metrologia. 41, S1-S15(2004).

4. B. Christian, Development and realisation of a fully automatic testing facility for determining the volume of $\mathrm{E}_{1}$ weights up to $50 \mathrm{~kg}$. in Fundamental and Applied Metrology (Lisbon, Portugal, 2009).

5. K. H. Chang and Y. J.Lee, Metrologia. 41, S95-S99(2004).

6. K. Riski and H.Kajastie, Meas. Sci. Technol. 15,1687-1693(2004). 